
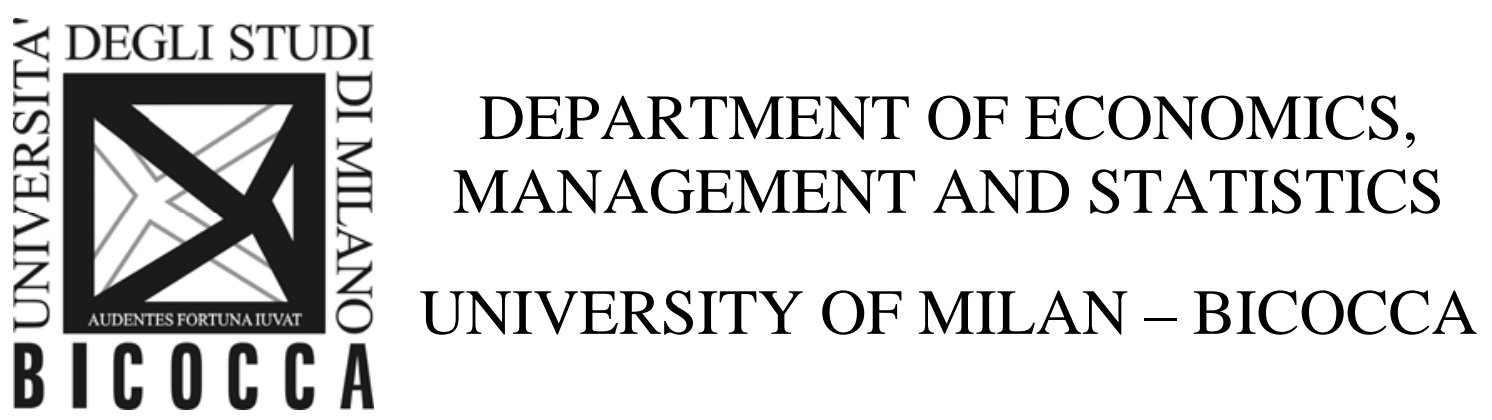

DEMS WORKING PAPER SERIES

\title{
Real and financial interacting oscillators: a behavioral macro-model with animal spirits
}

Ahmad Naimzada, Marina Pireddu

No. 268 - February 2014

Dipartimento di Economia, Metodi Quantitativi e Strategie di Impresa Università degli Studi di Milano - Bicocca

http://dems.unimib.it/ 


\title{
Real and financial interacting oscillators: a behavioral macro-model with animal spirits
}

\author{
Ahmad Naimzada ${ }^{\text {a* }}$, Marina Pireddu ${ }^{\text {b } \dagger}$ \\ ${ }^{a}$ Dept. of Economics, Quantitative Methods and Management, University of Milano-Bicocca, Milan \\ ${ }^{b}$ Dept. of Mathematics and Applications, University of Milano-Bicocca, Milan
}

\begin{abstract}
In this paper we propose a model in which the real side of the economy, described via a Keynesian good market approach, interacts with the stock market with heterogeneous speculators, i.e., optimist and pessimist fundamentalists. Employing analytical and numerical tools, we detect the mechanisms and the channels through which instabilities get transmitted between markets. In order to perform such analysis, we introduce the "interaction degree approach", which allows us to study the complete three-dimensional system by decomposing it into two subsystems, i.e., the isolated financial and real markets, easier to analyze, that are then interconnected through a parameter describing the interaction degree between the two markets. Next, we derive the stability conditions both for the isolated markets and for the whole system with interacting markets. Finally, we show how to apply the "interaction degree approach" to our model. To this aim, we first classify the possible scenarios according to the stability/instability of the isolated financial and real markets. For each of those frameworks we consider different possible parameter configurations and we show, both analytically and numerically, which are the effects of increasing the degree of interaction between the two markets. In particular, we find that the instability of the real market seems to have stronger destabilizing effects than the instability of the financial market: in fact, the former gets transmitted and possibly amplified by the connection with the financial market, while the latter gets dampened and possibly eliminated by the connection with the real market. We conclude our analysis by showing which are the effects of an increasing bias. Although it is clearly destabilizing when markets are isolated,
\end{abstract}

*Mailing address: University of Milano-Bicocca, U7 Building, Via Bicocca degli Arcimboldi 8, 20126 Milano, Italy. Tel.: +390264485813; fax: +390264483085. E-mail address: ahmad.naimzada@unimib.it

${ }^{\dagger}$ Corresponding author at: University of Milano-Bicocca, U5 Building, Via Cozzi 55, 20125 Milano, Italy. Tel.: +39 0264485767; fax: +390264485705. E-mail address: marina.pireddu@unimib.it 
its role becomes more ambiguous when the markets are interconnected. However, our numerical simulations suggest that increasing the bias has generally a destabilizing effect.

Keywords: nonlinearities; complex dynamics; oscillators; Keynesian model; animal spirits; behavioral finance.

JEL classification: C62, D84, E12, E32, G02

\section{Introduction}

Instabilities are known, both empirically and theoretically, to be features of all markets: the product markets, the labor market, and the financial markets. Over the last twenty years, many stock market models have been proposed in order to study the dynamics of financial markets (see De Grauwe, 2012 and Hommes, 2013). According to such models, even in the absence of stochastic shocks, the interaction between heterogeneous speculators accounts for the dynamics of financial markets. Those models, when endowed with stochastic shocks, are able to replicate some important phenomena, such as bubbles and crashes, excess volatility and volatility clustering (see, for instance, Brock and Hommes, 1997, 1998; Chiarella, 1992; Chiarella and He, 2002; De Grauwe and Grimaldi, 2006a, 2006b; Frankel and Froot, 1986, 1990; Lux and Marchesi, 1999). However, in this kind of models authors have restricted their attention to the representation and the dynamics of financial markets only and the existing feedbacks between the real and financial markets are often completely neglected. Some exceptions are represented, for instance, by Charpe et al. (2011), Lengnick and Wohltmann (2013), Scheffknecht and Geiger (2011) and Westerhoff (2012). Charpe et al. (2011) propose an integrated macro model, using a Tobin-like portfolio approach, and consider the interaction among heterogeneous agents in the financial market in order to generate financial market instability. They find that unorthodox fiscal and monetary policies are able to stabilize unstable macroeconomies. Lengnick and Wohltmann (2013) propose an agent-based model with financial markets interconnected with a New Keynesian model with bounded rationality and explore the consequences of transaction taxes. The results are endogenous developments of business cycles and stock price bubbles. Scheffknecht and Geiger (2011) present a financial market model with leverage-constrained heterogeneous agents integrated with a New Keynesian standard model; all agents are assumed to be boundedly rational. Those authors show that a systematic reaction by central bank on financial market developments dampens macroeconomic volatility. Finally, in Westerhoff (2012) the real economy is described via a Keynesian good market approach, while the set-up for the stock market includes heterogeneous speculators.

Inside the heterogeneous agent literature, only few papers deal with heterogeneous fundamentalists (see, for instance, De Grauwe and Rovira Kaltwasser, 2007; Diks and 
Dindo, 2008; Heitger, 2010; Manzan and Westerhoff, 2005; Naimzada and Ricchiuti, 2008, 2009; Rovira Kaltwasser, 2010; Westerhoff, 2003).

Our paper belongs both to the strand of literature on the interactions between real and financial markets, as well as to the literature with heterogeneous fundamentalists. In fact, we here present a model in which the real economy, described via a Keynesian good market approach, and the stock market, with heterogeneous speculators, interact. More precisely, similarly to De Grauwe and Rovira Kaltwasser (2012), we assume that the financial side is represented by a market where traders behave in two different ways: optimism and pessimism. On the other hand, in De Grauwe and Rovira Kaltwasser (2012) only the financial sector is considered, while the connection with the real side of the economy is missing. When comparing our setting to the one in Westerhoff (2012), we stress that, similarly to what done in that paper, we assume the real economy to be represented by an income-expenditure model in which expenditures depend also on the dynamics of the stock market price. On the other hand, in Westerhoff (2012) the real market subsystem is described by a stable linear relation, while the financial sector is represented by a nonlinear relation, that is, by a cubic functional relation. In that way, the oscillating behavior is generated by the financial subsystem only. In our paper we present instead a model in which the oscillating behavior is generated also by the real subsystem. To be more precise, the nonlinearity of the real subsystem is due to the nonlinearity of the adjustment mechanism of the good market with respect to the excess demand.

As regards the interaction between the two markets we assume that economic agents operating in the financial market base their decisions on a weighted average between an exogenous fundamental value and an endogenous fundamental value given by the current realization of income, while in the real market we assume that private expenditures depend also, with a given weight, on the stock market price. In particular, in our model the parameter describing the weights represents also the degree of interaction between the two markets. The extreme values of the weighting parameter correspond to the two cases of isolated markets and fully interacting markets, respectively.

Analytical and numerical tools are used in order to detect the mechanisms and the channels through which instabilities get transmitted between markets. The main contribution of this paper to the existing literature is in fact to focus on the role of real and financial feedback mechanisms, not only in relation to the dynamics and stability of a single market, but for those of the economy as a whole.

More precisely, we start by introducing the "interaction degree approach", which allows us to study high-dimensional systems with many parameters by decomposing them into subsystems easier to analyze, that are then interconnected through the "interaction parameter". Next, we introduce our model and we derive the stability conditions both for the isolated markets and for the whole system with interacting markets. In particular, we find that it is possible to isolate the parameter describing the degree of interaction between the two markets and that the stability conditions are fulfilled if it belongs to a range described by two lower bounds and two upper 
bounds. Finally, we show how to apply the "interaction degree approach" to our model. To this aim, we first classify the possible scenarios according to the stability/instability of the isolated financial and real markets: in this way we are led to analyze four frameworks. For each of those we consider different possible parameter configurations and we show, both analytically and numerically, which are the effects of increasing the degree of interaction between the two markets. The conclusions we get are not univocal: indeed, depending on the value of the other parameters, an increase in the interaction parameter may either have a stabilizing or a destabilizing effect, but also other phenomena are possible. Namely, according to the mutual position of the above mentioned lower and upper bounds of the stability range, it may also happen that the stabilization of the system occurs just for intermediate values of the interaction parameter, neither too small, nor too large, or it may as well happen that one of the the upper bounds is always negative or smaller than one of the lower bounds and thus we never get a complete stabilization of the system, even if its complexity may decrease and we observe some periodicity windows.

In more detail, the conclusions we get in the various scenarios are summarized hereinafter.

If both subsystems are stable when markets are isolated, the connection may either maintain the stability or have destabilizing effects, according to the parameter configuration considered. In particular, the market maker price adjustment parameter seems to play a crucial role in determining the behavior of the connected markets: indeed, increasing its value we observe a destabilization of the whole system.

If just the real subsystem is stable when markets are isolated, the connection reduces the complexity of the economy as a whole and may also lead to a complete stabilization. The parameter describing the real market speed of adjustment between demand and supply seems to play a crucial role in determining the behavior of the connected markets: indeed, increasing its value we observe a progressive reduction of the stability range for the interaction parameter, until the stable fixed point is replaced by a period-two orbit. We stress that in the existing literature on nonlinearities it is possible to find some examples of stabilization phenomena for interacting oscillating subsystems (see, for instance, Lloyd, 1995). In particular the stabilization is obtained by increasing the interaction degree between the subsystems. However, differently from our model, in those examples the subsystems are symmetric and described by the same functional relation: moreover, to the best of our knowledge, they do not belong to the economic literature, but they rather concern biological or physical systems.

If only the financial subsystem is stable when markets are isolated, the connection may reduce the complexity of the whole economy until determining the presence of period-two orbits, but we do not observe a complete stabilization. Again, the market maker price adjustment parameter seems to play a crucial role in determining the behavior of the connected markets: indeed, increasing its value we observe a further destabilization of the whole system, whose dynamics are characterized also by the 
presence of the so called "bubbles" (see Hommes 1991, 1994) and by chaotic attractors.

Finally, if both subsystems are unstable when markets are isolated, the connection may reduce the complexity of the whole economy until determining the presence of low-period orbits, alternating with chaotic bands, but we do not observe a complete stabilization.

Hence, the instability of the real market seems to have stronger destabilizing effects than the instability of the financial market: in fact, the former gets transmitted and possibly amplified by the connection with the financial market, while the latter gets dampened and possibly eliminated by the connection with the real market.

We conclude our analysis by showing which are the effects of an increasing bias. Although its effect is clearly destabilizing when markets are isolated, its role becomes more ambiguous when the markets are interconnected. Indeed, increasing the bias may have either a stabilizing or a destabilizing role, according to the value of the other parameters. However, it seems that increasing the bias has generally a destabilizing effect, as usually we do not reach a complete stabilization, or we achieve it just in small intervals for the corresponding parameter.

The remainder of the paper is organized as follows. In Section 2 we illustrate the approach we use to analyze the two interacting markets. In Section 3 we introduce the model, composed by the real and financial sectors. In Section 4 we derive the conditions for the steady state stability, both in the case of isolated and interacting markets. In Section 5 we classify and investigate, both analytically and numerically, the possible scenarios, determined by the stability/instability of the real and financial markets, when isolated. Finally, in Section 6 we draw some conclusions and discuss our results.

\section{The interaction degree approach}

As we shall see in Section 3, when considering both financial and real markets, we are led to analyze a nonlinear high-dimensional system with many parameters. Such features do not allow to easily handle that kind of systems from an analytical viewpoint when all the parameters vary, even if we are able to analytically determine the steady state and the stability conditions for the whole system. For this reason, we propose an approach that consists in studying, as a first step, the framework with isolated market, which have a lower dimensionality, are simpler to investigate and whose different behaviors can be easily classified. Then we make the parameter representing the interaction degree increase, keeping the other parameters fixed. In this way we are able to analytically find (if it exists) the set of values of the interaction parameter that implies stability. Moreover, the use of numerical tools allows us to understand what happens also in the unstable regime. This is in fact the strategy we are going to employ in Section 4 to classify the various scenarios for our system. However, in Section 3 we believe that the exposition is made more fluent by starting 
with the analysis of the stability conditions for the case of interacting markets and by deriving next the stability conditions for the framework with isolated markets.

In symbols, if we denote our integrated system by $S_{\omega}\left(x_{1}, \ldots, x_{N}\right)$, where $\omega \in$ $[0,1]$ is the interaction degree parameter, when setting $\omega=0$ we are led to study two (or, in general, more) isolated subsystems, we denote by $S_{0}^{1}\left(x_{1}, \ldots, x_{m}\right)$ and $S_{0}^{2}\left(x_{m+1}, \ldots, x_{N}\right)$, for some $m \in\{1, \ldots, N-1\}$. When instead $\omega=1$ the subsystems are fully integrated. The case with $\omega \in(0,1)$ represents a partial interaction between the subsystems.

In our framework, when setting $\omega=0$, we find two isolated subsystems, describing the financial and real markets, respectively. The former is described by two variables, the stock price and the difference between the shares of optimist and pessimist agents, while the latter is described by a unique variable, the level of income. We stress that the influence of the real market on the financial market is due to the fact that the reference value used in the decisional mechanism by the agents in the financial market is determined by the level of income. On the other hand, the investments depend also on the price of the financial asset. Such a double interaction is described by the parameter $\omega$.

\section{The model}

\subsection{The stock market}

With respect to the stock market, we assume that agents are not able to observe the true underlying fundamental. We suppose instead that they form believes about the fundamental and, on the basis of this belief, they operate in the stock market. We consider the trading behavior of two types of speculators: optimists and pessimists. The label optimist (pessimist) refers to traders that systematically overestimate (underestimate) the reference value used in their decisional mechanism. Both types of agents belong to the class of fundamentalists as, believing that stock prices will return to their fundamental value, they buy stocks in undervalued markets and sell stocks in overvalued markets ${ }^{1}$. Optimists and pessimists behave in a similar manner, a part from the fact that the beliefs they have about the reference value, we denote by $F_{t}^{o p t}$ and $F_{t}^{\text {pes }}$, differ. The perceived reference values are a weighted average between an exogenous value $\left(F^{*}+a\right.$ and $F^{*}-a$, respectively, with $\left.a>0\right)$ and a term depending on the income value. As regards the latter term, for simplicity, according to Naimzada and Pireddu (2013) and Westerhoff (2012), we assume for it a direct relationship with the economic activity value, both for optimists and pessimists. In particular, the endogenous term of the fundamental value perceived by optimists and pessimists is given by $k Y_{t}+a$ and $k Y_{t}-a$, respectively, where $Y_{t}$ is the national income and $k$

\footnotetext{
${ }^{1}$ To be more precise, we should say that we model agents as fundamentalists, but their effective behavior depends on the relative position of the stock price with respect to the perceived reference values (see (3.3) and (3.4)).
} 
is a positive parameter capturing the above described direct relationship. Hence, we assume that

$$
F_{t}^{o p t}=(1-\omega)\left(F^{*}+a\right)+\omega\left(k Y_{t}+a\right)=(1-\omega) F^{*}+\omega k Y_{t}+a
$$

and

$$
F_{t}^{\text {pes }}=(1-\omega)\left(F^{*}-a\right)+\omega\left(k Y_{t}-a\right)=(1-\omega) F^{*}+\omega k Y_{t}-a,
$$

where $a>0$ is the belief bias and $F^{*}$ is the true unobserved fundamental, both exogenously determined. The constant $\omega \in[0,1]$ represents the weighting average parameter. In particular, when $\omega=0$ the reference value is completely exogenous and coincides with the reference value of an isolated stock market like in De Grauwe and Rovira Kaltwasser (2012). When instead $\omega=1$ the reference value is endogenous. Optimists' demand is given by

$$
d_{t}^{o p t}=\alpha\left(F_{t}^{o p t}-P_{t}\right)
$$

and, similarly, pessimists' demand is given by

$$
d_{t}^{\text {pes }}=\alpha\left(F_{t}^{\text {pes }}-P_{t}\right),
$$

where $P_{t}$ is the stock price and $\alpha>0$ is the reactivity parameter.

The market maker determines excess demand and adjusts the stock price for the next period. In particular, we denote by $n_{t}^{i}, i \in\{o p t, p e s\}$, the fraction of traders of type $i$ in the market at time $t$ and we assume that the market maker behavior is described by the linear price adjustment mechanism

$$
P_{t+1}=P_{t}+\mu\left(n_{t}^{o p t} d_{t}^{o p t}+n_{t}^{\text {pes }} d_{t}^{\text {pes }}\right),
$$

where $\mu>0$ is the market maker price adjustment parameter. For simplicity, we normalize the population size to 1 . According to (3.5), the market maker increases (decreases) the stock price if excess demand $n_{t}^{\text {opt }} d_{t}^{\text {opt }}+n_{t}^{\text {pes }} d_{t}^{\text {pes }}$ is positive (negative). We set $x_{t}=n_{t}^{o p t}-n_{t}^{\text {pes }}$, in order to express the fraction of traders as $n_{t}^{o p t}=\frac{1+x_{t}}{2}$ and $n_{t}^{\text {pes }}=\frac{1-x_{t}}{2}$, so that we can rewrite (3.5) as

$$
P_{t+1}=P_{t}+\frac{\alpha \mu}{2}\left[\left(F_{t}^{o p t}-P_{t}\right)\left(1+x_{t}\right)+\left(F_{t}^{p e s}-P_{t}\right)\left(1-x_{t}\right)\right] .
$$

Recalling the definition of $F_{t}^{o p t}$ and $F_{t}^{\text {pes }}$ from (3.1) and (3.2), respectively, we rewrite (3.6) as

$$
P_{t+1}=P_{t}+\alpha \mu\left\{\left[(1-\omega) F^{*}+\omega k Y_{t}\right]-P_{t}+a x_{t}\right\} .
$$

We observe that the evolution of the stock price is determined by two factors. The first one is the deviation of the stock price from its unbiased reference value $\left(\left[(1-\omega) F^{*}+\omega k Y_{t}\right]-P_{t}\right)$ : when the price in period $t$ is below (above) its unbiased reference value, the price will increase (decrease) in the next period. The second 
factor involves the fraction of optimists and pessimists in the market. If $x_{t}$ is positive (negative) there are more (less) optimists than pessimists, so that the price will increase (decrease) in the next period. The strength of such effect is affected by the belief bias $a$. Finally, we notice that with a completely exogenous reference value, i.e., with $\omega=0$, and under rational expectations, (3.7) has a unique steady state given by $P^{*}=F^{*}$.

Defining now the dynamics of the population of traders, we will assume that they will start trying an optimistic or pessimistic behavior and, if it turns out to be the most profitable, they will stick to it; otherwise they will switch to the other behavior in the next period. Such an evolutionary process is governed by the profits that traders make in each period. Let us define the profits $\pi_{t}^{i}$ realized by type $i, i \in\{$ opt, pes $\}$, as

$$
\pi_{t}^{i}=d_{t-1}^{i}\left(P_{t}-P_{t-1}\right)
$$

Following Anderson et al. (1992) and Brock and Hommes (1997), we assume that the fraction $n_{t}^{i}$ of traders of type $i$ is given by the discrete choice model

$$
n_{t}^{i}=\frac{\exp \left(\beta \pi_{t}^{i}\right)}{\exp \left(\beta \pi_{t}^{o p t}\right)+\exp \left(\beta \pi_{t}^{p e s}\right)},
$$

where $\beta \geq 0$ is the parameter representing the intensity of choice. In particular, if $\beta=0$ the difference between profits is not considered and the behavior choice is purely random: hence, $n_{t}^{\text {opt }}=n_{t}^{\text {pes }}=\frac{1}{2}$. At the other extreme, when $\beta \rightarrow+\infty$, the switches are fully governed by the rational component and all traders are of the optimistic type $\left(x_{t} \rightarrow 1\right)$ if $\pi_{t}^{o p t}>\pi_{t}^{p e s}$, while all traders are of the pessimistic type $\left(x_{t} \rightarrow-1\right)$ if $\pi_{t}^{o p t}<\pi_{t}^{p e s}$; finally, if $\pi_{t}^{o p t}=\pi_{t}^{\text {pes }}$, we find again $n_{t}^{o p t}=n_{t}^{\text {pes }}=\frac{1}{2}$ and thus $x_{t}=0$.

From the definition of $x_{t}$ and (3.1)-(3.4), (3.7)-(3.9) it follows that

$$
x_{t}=\tanh \left(\frac{\beta\left(\pi_{t}^{o p t}-\pi_{t}^{p e s}\right)}{2}\right)=\tanh \left(\mu a \alpha^{2} \beta\left[(1-\omega) F^{*}+\omega k Y_{t-1}-P_{t-1}+a x_{t-1}\right]\right) .
$$

\subsection{The real market}

Similarly to Naimzada and Pireddu (2013) and Westerhoff (2012), we consider a Keynesian good market interacting with the stock market, in a closed economy with public intervention. It is assumed that private and government expenditures depend on national income and on the performance in the stock market. The dynamic behavior in the real economy is described by an adjustment mechanism depending on the excess demand. If aggregate excess demand is positive (negative), production will increase (decrease), that is, income $Y_{t+1}$ in period $t+1$ is defined in the following way

$$
Y_{t+1}=Y_{t}+\gamma g\left(Z_{t}-Y_{t}\right)
$$


where $g$ is an increasing function with $g(0)=0, Z_{t}$ is the aggregate demand in a closed economy, defined as

$$
Z_{t}=C_{t}+I_{t}+G_{t}
$$

where $C_{t}, I_{t}$ and $G_{t}$ stand for consumption, investment and government expenditure, respectively, and $\gamma>0$ is the real market speed of adjustment between demand and supply. In order to conduct our analysis, denoting by $E_{t}=Z_{t}-Y_{t}$ the excess demand, we specify the function $g$ as

$$
g\left(E_{t}\right)=a_{2}\left(\frac{a_{1}+a_{2}}{a_{1} e^{-E_{t}}+a_{2}}-1\right)
$$

with $a_{1}, a_{2}$ positive parameters.

With such choice, $g$ is increasing and $g(0)=0$. Moreover, it is bounded from below by $-a_{2}$ and from above by $a_{1}$. The presence of the two asymptotes prevents too large variations in income and thus prevents the real market from diverging, creating a real oscillator. We stress that this particular analytical specification does not compromise the generality of the results. In fact, we found analogous achievements for other sigmoid functions passing through the origin.

As commonly assumed, private and government expenditures increase with national income. Moreover, like in Naimzada and Pireddu (2013) and Westerhoff (2012), it is supposed that the financial situation of households and firms depends on the stock market performance, too. If the stock price increases, the same does private expenditure. On the basis of these considerations, we can write the relation between private and government expenditures and national income and stock price as

$$
Z_{t}=C_{t}+I_{t}+G_{t}=A+b Y_{t}+\omega c P_{t},
$$

where $A>0$ defines autonomous expenditure, $b \in[0,1]$ is the marginal propensity to consume and invest from current income, $c \in[0,1]$ is the marginal propensity to consume and invest from current stock market wealth, and $\omega \in[0,1]$ represents the degree of interaction between the real and the stock markets ${ }^{2}$. In particular, when $\omega=0$ the real market is completely isolated from the financial market; when $\omega=1$ the two markets are fully interconnected; for $\omega \in(0,1)$ we have a partial interaction. Inserting $Z_{t}$ from (3.12) into (3.10) and recalling the definition of $g$ in (3.11), we obtain the dynamic equation of the real market

$$
Y_{t+1}=Y_{t}+\gamma a_{2}\left(\frac{a_{1}+a_{2}}{a_{1} e^{-\left(A+b Y_{t}+\omega c P_{t}-Y_{t}\right)}+a_{2}}-1\right)
$$

\footnotetext{
${ }^{2}$ We stress that it would also be possible to assume that aggregate demand $Z_{t}$ depends, rather than on the stock price $P_{t}$, on the price variation $P_{t}-P_{t-1}$. Notice however that this would increase the dimensionality of our system. We will deal with such new formulation in a future paper.
} 
Summarizing, when taking into account both the financial and the real markets, we are led to study the following system describing the whole economy:

$$
\left\{\begin{array}{l}
P_{t+1}=P_{t}+\alpha \mu\left\{\left[(1-\omega) F^{*}+\omega k Y_{t}\right]-P_{t}+a x_{t}\right\} \\
x_{t+1}=\tanh \left(\mu a \alpha^{2} \beta\left[(1-\omega) F^{*}+\omega k Y_{t}-P_{t}+a x_{t}\right]\right) \\
Y_{t+1}=Y_{t}+\gamma a_{2}\left(\frac{a_{1}+a_{2}}{a_{1} e^{-\left(A+b Y_{t}+\omega c P_{t}-Y_{t}\right)}+a_{2}}-1\right)
\end{array}\right.
$$

The associated dynamical system is generated by the iterates of the three-dimensional map

$$
\begin{gathered}
G=\left(G_{1}, G_{2}, G_{3}\right):[0,+\infty) \times[-1,1] \times[0,+\infty) \rightarrow \mathbb{R}^{3}, \\
(P, x, Y) \mapsto\left(G_{1}(P, x, Y), G_{2}(P, x, Y), G_{3}(P, x, Y)\right),
\end{gathered}
$$

defined as:

$$
\left\{\begin{array}{l}
G_{1}(P, x, Y)=P+\alpha \mu\left((1-\omega) F^{*}+\omega k Y-P+a x\right) \\
G_{2}(P, x, Y)=\tanh \left(\mu a \alpha^{2} \beta\left[(1-\omega) F^{*}+\omega k Y-P+a x\right]\right) \\
G_{3}(P, x, Y)=Y+\gamma a_{2}\left(\frac{a_{1}+a_{2}}{a_{1} e^{-[A+b Y+\omega c P-Y]}+a_{2}}-1\right)
\end{array}\right.
$$

\section{Some local stability results}

In order to classify in Section 5 the various scenarios occurring for $\omega=0$ and investigate their local stability when $\omega$ increases, hereinafter we will derive the corresponding sufficient conditions both in the case of interacting and isolated markets. In fact, the classification we chose to adopt in the next section relies on the stability/instability features of the real and financial subsystems when they are isolated. Then, for any such scenario, we will study what happens when the interconnection between the two markets increases.

Straightforward computations show that there is a perfect correspondence between the numerical results in the next section and the analytical conditions derived in Subsections 4.1 and 4.2. In fact, for the reader's convenience, in correspondence to any scenario considered in Section 5 we will check what the analytical conditions say and we will compare those conditions with the numerical simulations performed therein.

\subsection{Interacting markets}

The map in (3.14) has a unique fixed point in

$$
\left(P^{*}, x^{*}, Y^{*}\right)=\left(\frac{\omega A k+(1-\omega) F^{*}(1-b)}{1-b-\omega^{2} c k}, 0, \frac{A+\omega c(1-\omega) F^{*}}{1-b-\omega^{2} c k}\right) .
$$

The Jacobian matrix for $G$ computed in correspondence to it reads as 


$$
J_{G}\left(P^{*}, x^{*}, Y^{*}\right)=\left[\begin{array}{lll}
1-\alpha \mu & \alpha \mu a & \alpha \mu \omega k \\
-\mu a \alpha^{2} \beta & \alpha^{2} \mu a^{2} \beta & \alpha^{2} \mu a \beta \omega k \\
\frac{\gamma a_{1} a_{2} \omega c}{a_{1}+a_{2}} & 0 & 1-\frac{\gamma a_{1} a_{2}(1-b)}{a_{1}+a_{2}}
\end{array}\right] .
$$

In order to check the stability of the steady state in the various scenarios considered in Section 4, we are going to use the following conditions (see Farebrother, 1973):

(i) $1+C_{1}+C_{2}+C_{3}>0$;

(ii) $1-C_{1}+C_{2}-C_{3}>0$;

(iii) $1-C_{2}+C_{1} C_{3}-\left(C_{3}\right)^{2}>0$;

(iv) $3-C_{2}>0$,

where $C_{i}, i \in\{1,2,3\}$, are the coefficients of the characteristic polynomial

$$
\lambda^{3}+C_{1} \lambda^{2}+C_{2} \lambda+C_{3}=0 .
$$

In our framework, we have

$$
\begin{aligned}
& C_{1}=\frac{\gamma a_{1} a_{2}(1-b)}{a_{1}+a_{2}}-2+\alpha \mu-\mu a^{2} \alpha^{2} \beta ; \\
& C_{2}=2 \mu a^{2} \alpha^{2} \beta+1-\alpha \mu-\frac{\gamma a_{1} a_{2} \omega^{2} c k \alpha \mu}{a_{1}+a_{2}}-\frac{\gamma a_{1} a_{2}(1-b)}{a_{1}+a_{2}}\left(1-\alpha \mu+\mu a^{2} \alpha^{2} \beta\right) ; \\
& C_{3}=\mu a^{2} \alpha^{2} \beta\left(\frac{\gamma a_{1} a_{2}(1-b)}{a_{1}+a_{2}}-1\right) .
\end{aligned}
$$

Notice that, making $\omega$ explicit, it is possible to rewrite Conditions (i)-(iv) above respectively as follows:

(i') $\omega^{2}<\left(1+C_{1}+\widetilde{C}+C_{3}\right) \frac{a_{1}+a_{2}}{\gamma a_{1} a_{2} c k \alpha \mu}:=B_{1}$;

(ii') $\omega^{2}<\left(1-C_{1}+\widetilde{C}-C_{3}\right) \frac{a_{1}+a_{2}}{\gamma a_{1} a_{2} c k \alpha \mu}:=B_{2}$;

(iii') $\omega^{2}>\left(-1+\widetilde{C}-C_{1} C_{3}+C_{3}^{2}\right) \frac{a_{1}+a_{2}}{\gamma a_{1} a_{2} c k \alpha \mu}:=B_{3}$;

(iv') $\omega^{2}>(\widetilde{C}-3) \frac{a_{1}+a_{2}}{\gamma a_{1} a_{2} c k \alpha \mu}:=B_{4}$,

where we have set

$$
\widetilde{C}=2 \mu a^{2} \alpha^{2} \beta+1-\alpha \mu-\frac{\gamma a_{1} a_{2}(1-b)}{a_{1}+a_{2}}\left(1-\alpha \mu+\mu a^{2} \alpha^{2} \beta\right) .
$$

Hence, if 
$\min \left\{1+C_{1}+\widetilde{C}+C_{3}, 1-C_{1}+\widetilde{C}-C_{3}\right\}>0$ and $\max \left\{-1+\widetilde{C}-C_{1} C_{3}+C_{3}^{2}, \widetilde{C}-3\right\}<1$, then the integrated system is locally asymptotically stable at the steady state if

$$
\max \left\{B_{3}, B_{4}\right\}<\omega^{2}<\min \left\{B_{1}, B_{2}\right\}, \omega \in[0,1]
$$

If instead

$\min \left\{1+C_{1}+\widetilde{C}+C_{3}, 1-C_{1}+\widetilde{C}-C_{3}\right\} \leq 0$ or $\max \left\{-1+\widetilde{C}-C_{1} C_{3}+C_{3}{ }^{2}, \widetilde{C}-3\right\} \geq 1$, it is not possible to have local stability at the steady state, for any $\omega \in[0,1]$.

\subsection{Isolated markets}

In the special case in which $\omega=0$, System (3.13) can be rewritten as

$$
\left\{\begin{array}{l}
P_{t+1}=P+\alpha \mu\left(F^{*}-P+a x\right) \\
x_{t+1}=\tanh \left(\mu a \alpha^{2} \beta\left[F^{*}-P+a x\right]\right) \\
Y_{t+1}=Y+\gamma a_{2}\left(\frac{a_{1}+a_{2}}{a_{1} e^{-[A+(b-1) Y]}+a_{2}}-1\right)
\end{array}\right.
$$

and its steady state reads as

$$
\left(P^{*}, x^{*}, Y^{*}\right)=\left(F^{*}, 0, \frac{A}{1-b}\right) .
$$

Since in such framework the first two equations in (4.3) depend only on $P$ and $x$, and the last one only on $Y$, implying that the real and stock markets are not related, as explained in Section 2, instead of considering the three-dimensional system, we will deal with the two-dimensional subsystem related to the stock market

$$
\left\{\begin{array}{l}
P_{t+1}=P_{t}+\alpha \mu\left\{F^{*}-P_{t}+a x_{t}\right\} \\
x_{t+1}=\tanh \left(\mu a \alpha^{2} \beta\left[F^{*}-P_{t}+a x_{t}\right]\right)
\end{array}\right.
$$

and the one-dimensional subsystem related to the real market

$$
Y_{t+1}=Y_{t}+\gamma a_{2}\left(\frac{a_{1}+a_{2}}{a_{1} e^{-\left(A+(b-1) Y_{t}\right)}+a_{2}}-1\right)
$$

In this way, in agreement with the findings in De Grauwe and Rovira Kaltwasser (2012), the steady state above should be split as

$$
\left(P^{*}, x^{*}\right)=\left(F^{*}, 0\right), \quad Y^{*}=\frac{A}{1-b}
$$


and, similarly, the Jacobian matrix in (4.1) computed in correspondence to the steady state should be split as

$$
J^{1}\left(P^{*}, x^{*}\right)=\left[\begin{array}{ll}
1-\alpha \mu & \alpha \mu a \\
-\mu a \alpha^{2} \beta & \alpha^{2} \mu a^{2} \beta
\end{array}\right], \quad J^{2}\left(Y^{*}\right)=1-\frac{\gamma a_{1} a_{2}(1-b)}{a_{1}+a_{2}} .
$$

The Jury conditions for the financial subsystem read as

$$
\begin{aligned}
& \operatorname{det} J^{1}=\mu \alpha^{2} a^{2} \beta<1, \\
& 1+\operatorname{tr} J^{1}+\operatorname{det} J^{1}=2-\mu \alpha+2 \mu \alpha^{2} a^{2} \beta>0, \\
& 1-\operatorname{tr} J^{1}+\operatorname{det} J^{1}=\mu \alpha>0 .
\end{aligned}
$$

Notice that the third condition is always fulfilled, while the first two can be rewritten, making $\beta$ explicit, as

$$
\frac{\alpha \mu-2}{2 \mu \alpha^{2} a^{2}}<\beta<\frac{1}{\mu \alpha^{2} a^{2}}
$$

From (4.4) we easily infer the destabilizing role of the bias for the financial side of the economy when the two markets are isolated: indeed, the stability interval for $\beta$ gets reduced when $a$ increases.

On the other hand, the real subsystem is locally asymptotically stable at the steady state if $-1<1-\frac{\gamma a_{1} a_{2}(1-b)}{a_{1}+a_{2}}<1$. The right inequality is always fulfilled (except for $b=1$, but we will always deal with the case $0<b<1$ ), while the left inequality holds if and only if

$$
\gamma<\frac{2\left(a_{1}+a_{2}\right)}{a_{1} a_{2}(1-b)}
$$

Hence, when $\omega=0$ both subsystems are stable if

$$
\frac{\alpha \mu-2}{2 \mu \alpha^{2} a^{2}}<\beta<\frac{1}{\mu \alpha^{2} a^{2}} \quad \text { and } \quad \gamma<\frac{2\left(a_{1}+a_{2}\right)}{a_{1} a_{2}(1-b)}
$$

\section{Possible scenarios}

Starting from the various stability/instability scenarios for the financial and real subsystems when isolated, in the next pages we shall investigate what happens in each framework when the degree of interaction between the two markets increases, in order to show that varying the parameter $\omega$ may produce very different effects depending on the value of the other parameters and the specific framework considered.

We conclude the section by analyzing the effects of an increasing bias on the stability of the whole system. 


\subsection{Stable financial and real subsystems}

In this framework, when isolated, both markets are stable. When $\omega$ increases, the steady state can either remain stable until $\omega=1$ or can undergo a flip bifurcation, followed by a secondary double Neimark-Sacker bifurcation, according to the considered value of the parameters. In particular, the parameter $\mu$ seems to play a crucial role in this respect. In fact, in Figures 1-3 below we have fixed the parameters as follows: $F^{*}=5, k=0.25, \alpha=0.08, \beta=1, c=1, a=2, \gamma=3.5, a_{1}=2, a_{2}=4, A=5, b=$ 0.7 , and $\mu=5$ in Figure 1, while $\mu=28$ in Figures 2 and 3. In Figure 1 the steady state remains stable until $\omega=1$, while in Figures 2 and 3 a destabilization occurs for $\omega \simeq 0.515$. More precisely, in Figures 1 and $2(\mathrm{~A})$ we show the bifurcation diagram for $P$ with respect to $\omega \in[0,1]$, while in Figure $2(\mathrm{~B})$ we show the bifurcation diagram with respect to $\omega \in[0,1]$ for $Y$; in Figure 2 (C) we show the Lyapunov exponent when $\omega$ varies in $[0,1]$. In Figures $3(\mathrm{~A})$ and $(\mathrm{B})$ we depict, in the phase plane, the fixed point when $\omega=0.25$ and the period-two cycle when $\omega=0.70$, respectively; finally, in Figure $3(\mathrm{C})$ we show the time series for $P$ (in red) and $Y$ (in blue) when $\omega=0.95$, which highlight a quasiperiodic behavior characterized by long monotonic increasing motions, followed by oscillatory decreasing motions.

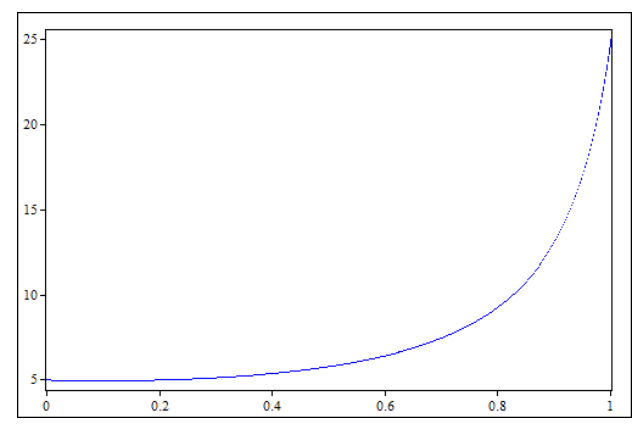

Figure 1: The bifurcation diagram with respect to $\omega \in[0,1]$ for $P$, for $\mu=5$ and the initial conditions $P(0)=5, x(0)=0.8$ and $Y(0)=25$. 


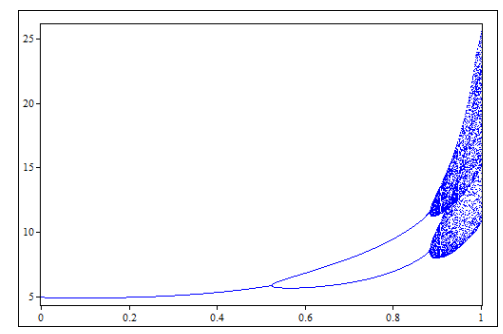

(A)

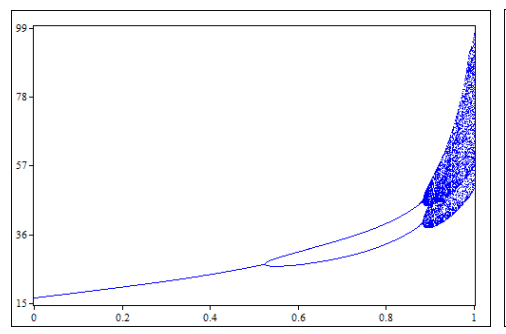

(B)

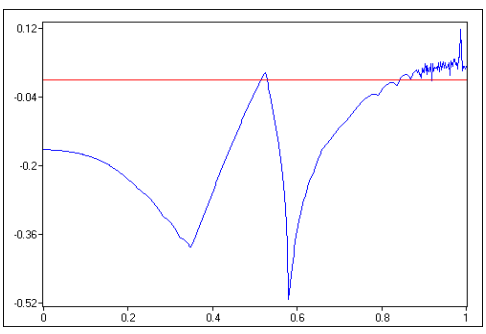

(C)

Figure 2: The bifurcation diagrams with respect to $\omega \in[0,1]$ for $\mathrm{P}$ in $(\mathrm{A})$ and $\mathrm{Y}$ in (B), and the Lyapunov exponent in $(\mathrm{C})$, respectively, for $\mu=28$ and the initial conditions $P(0)=5, x(0)=0.8$ and $Y(0)=25$.

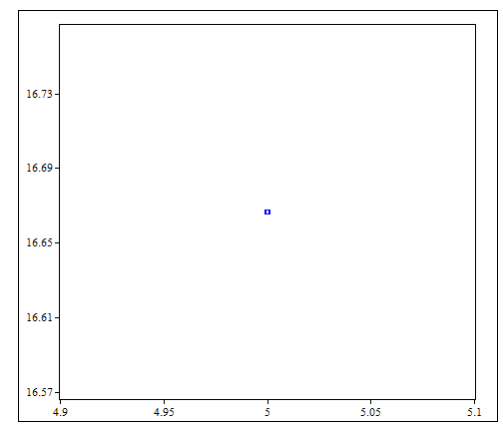

(A)

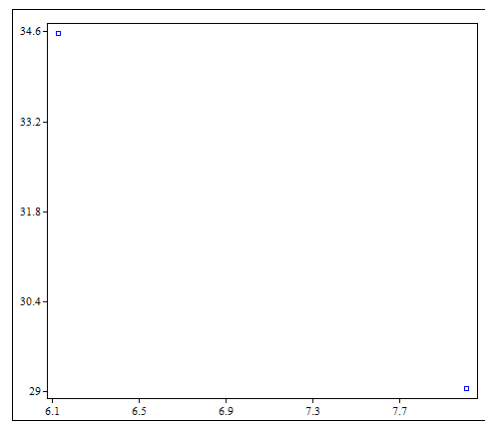

(B)

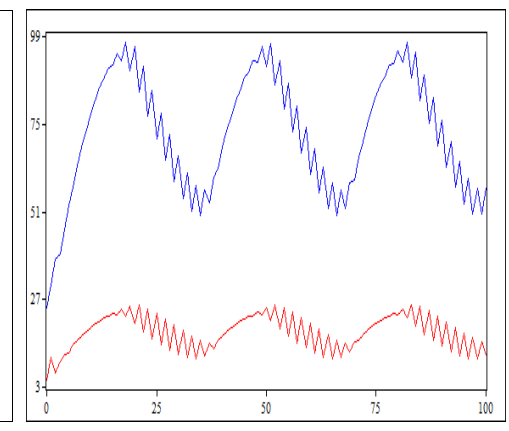

(C)

Figure 3: The $(P, Y)$-phase portraits for $\mu=28$, and $\omega=0.25$ in $(\mathrm{A})$ and $\omega=0.70$ in (B); in (C) the time series for $P$ in red (below) and $Y$ in blue (above) when $\mu=28$ and $\omega=0.95$.

Let us now check whether the theoretical results in Section 4 are in agreement with the numerical achievements above.

First of all let us verify that, for the choice of both the parameter sets above, when $\omega=0$ both the financial and real subsystems are stable, i.e., let us check that all the inequalities in (4.5) are fulfilled. A straightforward computation shows that this is the case, as the first chain of inequalities reads as $-6.25<1<7.812$ and the last inequality becomes $3.5<5$ when $\mu=5$, while the first chain of inequalities reads as $0.167<1<1.395$ and the last inequality is again $3.5<5$ when $\mu=28$.

As concerns the stability conditions when $\omega$ varies in $[0,1]$, when $\mu=5$ we have $B_{1}=1.2, B_{2}=2.386, B_{3}=-2.4509, B_{4}=-6.778$, and thus (4.2) reads as $\omega \in[0,1]$, that is, the system is stable for any $\omega$, in agreement with Figure 1 ; when instead $\mu=28$ we have $B_{1}=1.2, B_{2}=0.274, B_{3}=-0.098, B_{4}=-0.793$, and thus (4.2) reads as $\omega \in\left[0, \sqrt{B_{2}}\right)=[0,0.523)$, that is, the system is stable just for small values of $\omega$, in agreement with Figure 2. 
Hence, what we find in this scenario is that increasing $\mu$ has a destabilizing effect. In fact, fixing all the other parameters as above, and letting just $\mu$ vary, we find that $\frac{\partial B_{1}}{\partial \mu}=0, \frac{\partial B_{2}}{\partial \mu}<0, \frac{\partial B_{3}}{\partial \mu}>0$ and $\frac{\partial B_{4}}{\partial \mu}>0$. Hence, the stability region decreases when $\mu$ increases (as $B_{1}$ does not vary with $\mu$ and the upper bound $B_{2}$ decreases, while the lower bounds $B_{3}$ and $B_{4}$ increase), confirming the highlighted destabilizing effect of the parameter $\mu$.

Summarizing, for the above parameter configurations, the interaction between the financial and real markets either maintains the stability of the system, or it has a destabilizing effect, through a flip bifurcation. We stress that, differently from De Grauwe and Rovira Kaltwasser (2012), in our model after the flip bifurcation we have a persistent regime characterized by a two-cycle; moreover, for larger values of $\omega$ we find quasiperiodic motions, following a secondary double Neimark-Sacker bifurcation.

\subsection{Unstable financial subsystem - stable real subsystem}

In the framework we are going to consider, when isolated, the financial subsystem is unstable and characterized by quasiperiodic motions, while the real subsystem is stable. For not too large values of the parameter $\gamma$, when $\omega$ increases, a stable fixed point emerges through a Neimark-Sacker bifurcation. According to the value of $\gamma$, that fixed point can either persist until $\omega=1$ or can undergo a flip bifurcation and then a secondary double Neimark-Sacker bifurcation; for even larger values of $\gamma$, we just obtain a reduction of the complexity of the system for suitable intermediate values of $\omega$, but the system is never stabilized.

More precisely, in Figures 4-6 below, the corresponding parameters are: $F^{*}=2, k=$ $0.1, \alpha=0.08, \beta=1, c=1, a=2.4, \mu=28, a_{1}=3, a_{2}=1, A=12, b=0.7$, and $\gamma=5$ in Figure $4, \gamma=8$ in Figure 5, and $\gamma=8.8$ in Figure 6. In Figure 4 the fixed point becomes stable for $\omega \simeq 0.2$ and remains stable until $\omega=1$. In Figure 5, instead of remaining stable, it undergoes a flip bifurcation for $\omega \simeq 0.5$ and then a secondary double Neimark-Sacker bifurcation for $\omega \simeq 0.96$. In Figure 6 the fixed point is never stable: we just observe a reduction of the complexity of the system for $\omega \in(0.2,0.8)$, where we have a stable period-two cycle. In more details, in Figures $4(\mathrm{~A}), 5$ (A) and $6(\mathrm{~A})$ we show the bifurcation diagrams with respect to $\omega \in[0,1]$ for $P$, while in Figures 4 (B), 5 (B) and 6 (B) we show the bifurcation diagrams for $P$; in Figures 4 $(\mathrm{C}), 5(\mathrm{C})$ and $6(\mathrm{C})$ we show the Lyapunov exponents when $\omega$ varies in $[0,1]$. 


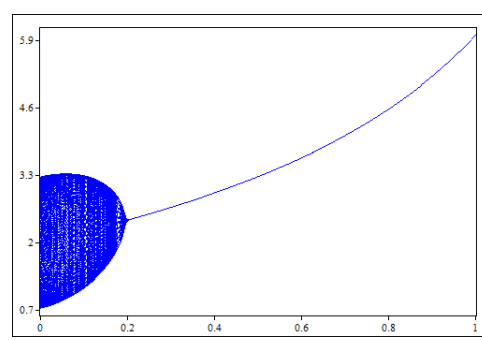

(A)

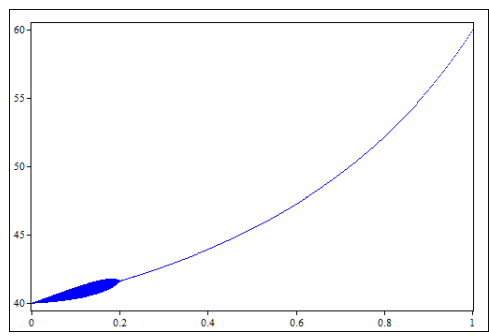

(B)

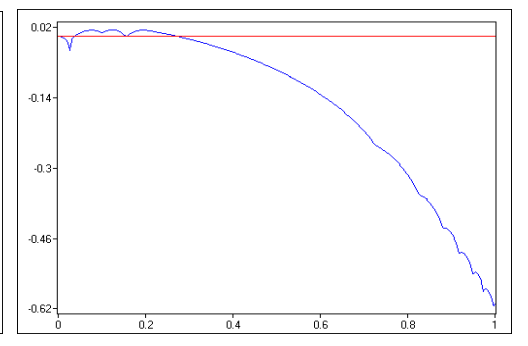

(C)

Figure 4: The bifurcation diagrams with respect to $\omega \in[0,1]$ for $P$ in $(\mathrm{A})$ and $Y$ in $(\mathrm{B})$, and the Lyapunov exponent in $(\mathrm{C})$, respectively, for $\gamma=5$ and the initial conditions $P(0)=12, x(0)=-0.3$ and $Y(0)=61$.

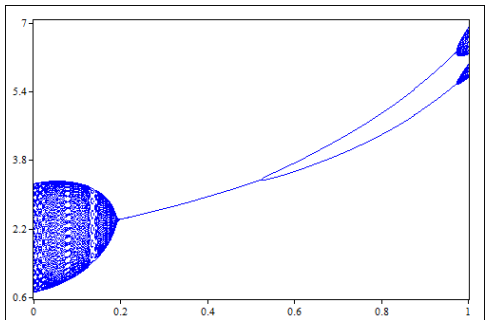

(A)

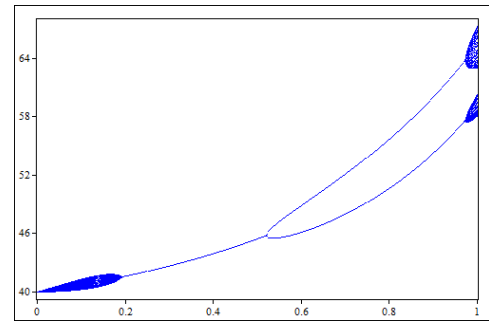

(B)

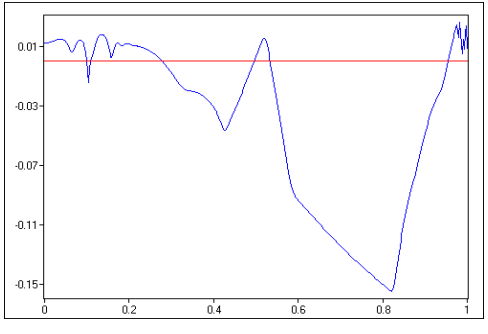

(C)

Figure 5: The bifurcation diagrams with respect to $\omega \in[0,1]$ for $P$ in $(\mathrm{A})$ and $Y$ in (B), and the Lyapunov exponent in $(\mathrm{C})$, respectively, for $\gamma=8$ and the initial conditions $P(0)=12, x(0)=-0.3$ and $Y(0)=61$.

Let us now check whether the theoretical results in Section 4 are in agreement with the numerical achievements above.

First of all let us verify that, for the choice of all the parameter sets above, when $\omega=0$ just the real subsystem is stable, i.e., let us check that the last inequality in (4.5) is fulfilled, but not the first chain of inequalities therein. A straightforward computation shows that this is the case, as the first chain of inequalities reads as $0.116<1<0.968$ and the last inequality becomes $5<8.888$ when $\gamma=5$; the first chain of inequalities reads again as $0.116<1<0.968$ and the last inequality is $8<8.888$ when $\gamma=8$; finally, the first chain of inequalities reads once again as $0.116<1<0.968$ and the last inequality is $8.8<8.888$ when $\gamma=8.8$.

As concerns the stability conditions when $\omega$ varies in $[0,1]$, when $\gamma=5$ we have $B_{1}=3, B_{2}=1.9004, B_{3}=0.0379, B_{4}=-2.3117$, and thus (4.2) reads as $\omega \in$ $\left(\sqrt{B_{3}}, 1\right]=(0.194,1]$, that is, the system is stable for large values of $\omega$, in agreement with Figure 4 ; when $\gamma=8$ we have $B_{1}=3, B_{2}=0.271, B_{3}=0.035, B_{4}=-1.34$, and thus (4.2) reads as $\omega \in\left(\sqrt{B_{3}}, \sqrt{B_{2}}\right)=(0.189,0.521)$, that is, the system is stable just for intermediate values of $\omega$, neither too small, nor too large, in agreement with Figure 


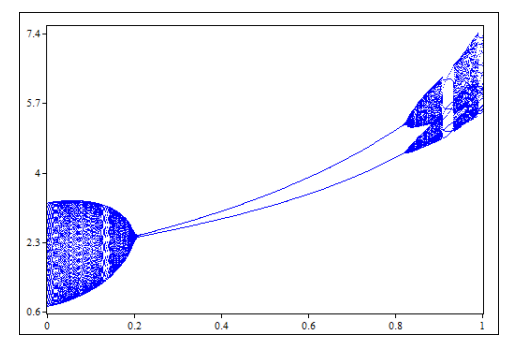

(A)

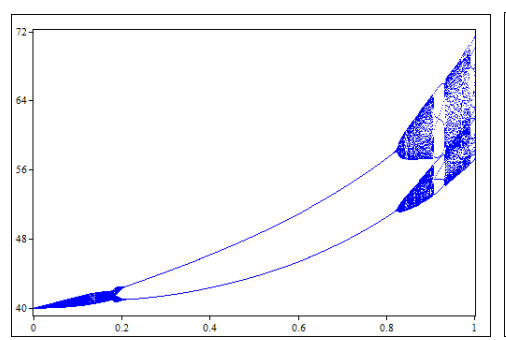

(B)

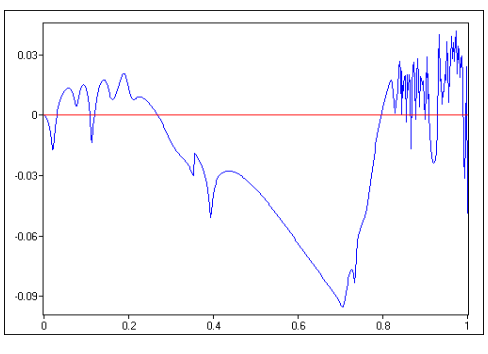

(C)

Figure 6: The bifurcation diagrams with respect to $\omega \in[0,1]$ for $P$ in (A) and $Y$ in (B), and the Lyapunov exponent in (C), respectively, for $\gamma=8.8$ and the initial conditions $P(0)=12, x(0)=-0.3$ and $Y(0)=61$.

5; finally, when $\gamma=8.8$ we have $B_{1}=3, B_{2}=0.024, B_{3}=0.038, B_{4}=-1.193$, and thus, since $B_{2}<B_{3}$, (4.2) implies that there exists no $\omega$ for which the system is stable, in agreement with Figure 6.

Hence, what we find is that increasing $\gamma$ has a destabilizing effect. In fact, fixing all the other parameters as above, and letting just $\gamma$ vary, we find that $\frac{\partial B_{1}}{\partial \gamma}=0$, $\frac{\partial B_{2}}{\partial \gamma}<0, \frac{\partial B_{3}}{\partial \gamma}$ changes sign (in particular, it is negative for $\gamma=5$ and positive for $\gamma=8$ and $\gamma=8.8)$, but $B_{3}$ always lies in $(0,0.1)$ for $\gamma \in[5,8.8]$ and thus it does not restrict the stability region too much, and finally $\frac{\partial B_{4}}{\partial \mu}>0$. Hence, the stability region decreases when $\gamma$ increases (as $B_{1}$ does not vary with $\gamma$ and the upper bound $B_{2}$ decreases, while $B_{3}$ is small and it does not vary a lot, and the lower bound $B_{4}$ increases), confirming the highlighted destabilizing effect of $\gamma$.

Summarizing, for the above parameter configurations, for small values of $\omega$, the instability of the financial market gets transmitted to the real market. However, increasing $\omega$ decreases the complexity of the whole system. This effect may either persist until $\omega=1$ or it may end for larger values of $\omega$, where we find instead quasiperiodic motions, following a secondary double Neimark-Sacker bifurcation of the period-two cycle. 


\subsection{Stable financial subsystem - unstable real subsystem}

In this framework, when isolated, the financial subsystem is stable, while the real subsystem is unstable. When $\omega$ increases, different possible behaviors may arise. We depict some of them in Figures 7-10 below, where we have fixed the parameters as follows: $F^{*}=2, k=0.1, \alpha=0.08, \beta=0.5, c=1, a=2.4, \gamma=11.5, a_{1}=3, a_{2}=$ 1, $A=12, b=0.7$, while we have $\mu=20$ in Figure $7, \mu=28$ in Figure 8 and $\mu=31$ in Figures 9 and 10.

More precisely, in Figure 7 (A) we show the bifurcation diagram with respect to $\omega \in[0.7,1]$ for $Y$, when $\mu=20$. For such parameter configuration, when $\omega \in[0,0.752]$ we have just a stable period-two cycle; at $\omega=0.752$ a period-six cycle emerges, which coexists with the period-two cycle (see the $(P, Y)$-phase portrait in Figure 7 (B) for $\omega=0.7948$ ). For $\omega \simeq 0.823$, through a double Neimark-Sacker bifurcation of the period-two cycle, quasiperiodic motions emerge, which coexist with the period-six cycle (see the $(P, Y)$-phase portrait in Figure $7(\mathrm{C})$ for $\omega=0.826$ ). For $\omega \simeq 0.830$, the quasiperiodic motions ends and only the period-six cycle survives until $\omega \simeq 0.88$, where quasiperiodic motions emerge, lasting until $\omega=1$.

In Figures $8(\mathrm{~A})$ and $9(\mathrm{~A})$, we show the bifurcation diagrams with respect to $\omega \in[0,1]$ for $P$, while in Figures $8(\mathrm{~B})$ and $9(\mathrm{~B})$, we show the bifurcation diagrams with respect to $\omega \in[0,1]$ for $Y$; in Figures $8(\mathrm{C})$ and $9(\mathrm{C})$, we represent the Lyapunov exponents when $\omega$ varies in $[0,1]$. Finally, in regard to the parameter configuration considered in Figure 9, we show in Figure $10(\mathrm{~A})$ the $(P, Y)$-phase portrait for $\omega=0.1$, where we have a period-two cycle, in Figure $10(\mathrm{~B})$ the $(P, Y)$-phase portrait for $\omega=0.4$, where we have a period-eight cycle, in Figure $10(\mathrm{C})$ the $(P, Y)$-phase portrait for $\omega=0.7$, where we have again a period-two cycle, and in Figure $10(\mathrm{D})$ the $(P, Y)$ phase portrait for $\omega=0.9$, where we have a chaotic attractor. 


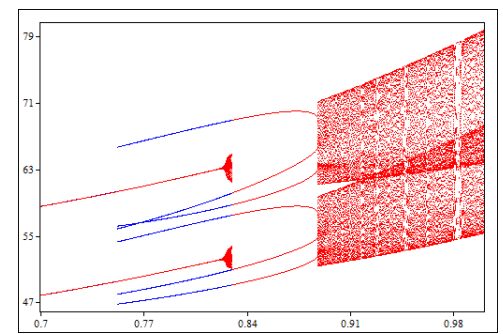

(A)

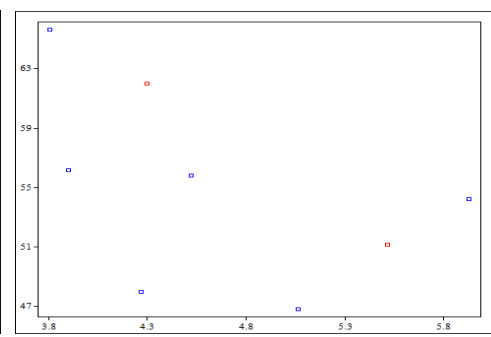

(B)

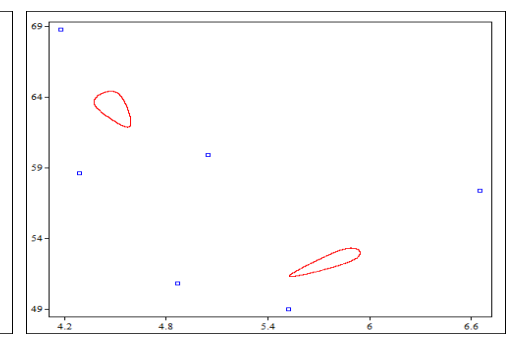

(C)

Figure 7: The bifurcation diagram with respect to $\omega \in[0.7,1]$ for $Y$ in $(\mathrm{A})$, for $\mu=20$ and the initial conditions $P(0)=\ldots, x(0)=\ldots$ and $Y(0)=\ldots$; the $(P, Y)$ phase portrait showing the coexistence of the period-six cycle with a period-two cycle for $\omega=0.7948$ in $(\mathrm{B})$, for $\mu=20$ and the initial conditions $P(0)=5, x(0)=0.3$ and $Y(0)=65$ for the period-two cycle and $P(0)=4, x(0)=0.3$ and $Y(0)=65$ for the period-six cycle; the $(P, Y)$-phase portrait showing the coexistence of the period-six cycle with two closed invariant curves for $\omega=0.826$ in $(\mathrm{C})$, for $\mu=20$ and the initial conditions $P(0)=4, x(0)=0.3$ and $Y(0)=65$ for the period-six cycle and $P(0)=4, x(0)=0.1$ and $Y(0)=64$ for the invariant curves.

Let us now check whether the theoretical results in Section 4 are in agreement with the numerical achievements above.

First of all let us verify that, for the choice of all the parameter sets above, when $\omega=0$ just the financial subsystem is stable, i.e., let us check that the first chain of inequalities in (4.5) is fulfilled, but not the last inequality therein. A straightforward computation shows that this is the case, as the first chain of inequalities reads as $-0.271<0.5<1.356$ and the last inequality becomes $11.5<8.888$ when $\mu=20$; the first chain of inequalities reads as $0.116<0.5<0.968$ and the last inequality is again $11.5<8.888$ when $\mu=28$; finally, the first chain of inequalities reads as $0.210<0.5<0.875$ and the last inequality is once again $11.5<8.888$ when $\mu=31$.

As concerns the stability conditions when $\omega$ varies in $[0,1]$, when $\mu=20$ we have $B_{1}=3, B_{2}=-0.484, B_{3}=-0.714, B_{4}=-1.640$, and thus, since $B_{2}<0$, (4.2) implies that there exists no $\omega$ for which the system is stable, in agreement with Figure 7 ; when $\mu=28$ we have $B_{1}=3, B_{2}=-0.240, B_{3}=-0.288, B_{4}=-0.690$, and thus, since again $B_{2}<0,(4.2)$ implies that there is no $\omega$ for which the system is stable, in agreement with Figure 8; finally, when $\mu=31$ we have $B_{1}=3, B_{2}=-0.182, B_{3}=$ $-0.199, B_{4}=-0.461$, and thus, since one again $B_{2}<0$, (4.2) implies that there is no $\omega$ for which the system is stable, in agreement with Figure 9.

Hence, for the selected parameter values, the system is never stable. From the pictures above we also notice that increasing $\mu$ has a further destabilizing effect, as the complexity of the system increases when $\mu$ moves from 20 to 31. As shown in Figures 9 and 10, in this latter case we have chaotic dynamics for values of $\omega$ close to 1. 


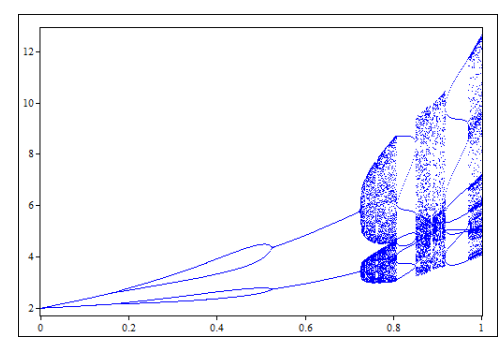

(A)

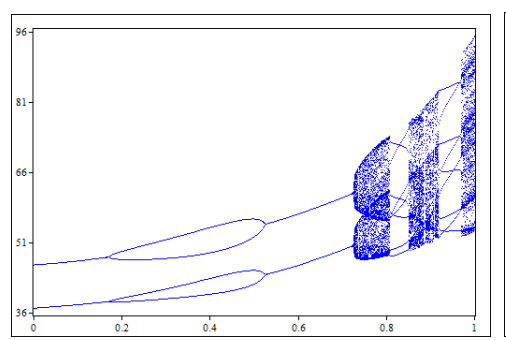

(B)

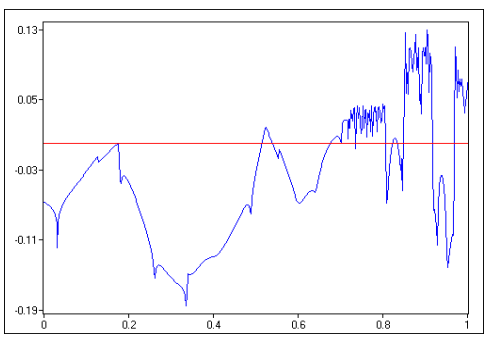

(C)

Figure 8: The bifurcation diagrams with respect to $\omega \in[0,1]$ for $P$ in $(\mathrm{A})$ and $Y$ in $(\mathrm{B})$, and the Lyapunov exponent in $(\mathrm{C})$, respectively, for $\mu=28$ and the initial conditions $P(0)=10, x(0)=0.5$ and $Y(0)=61$.

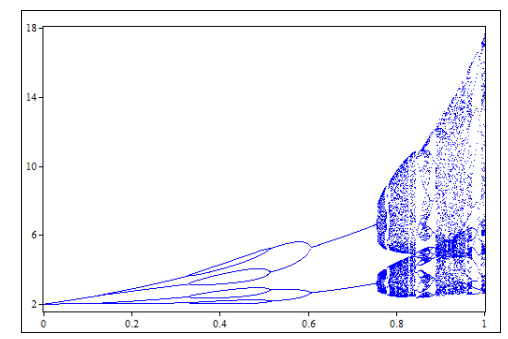

(A)

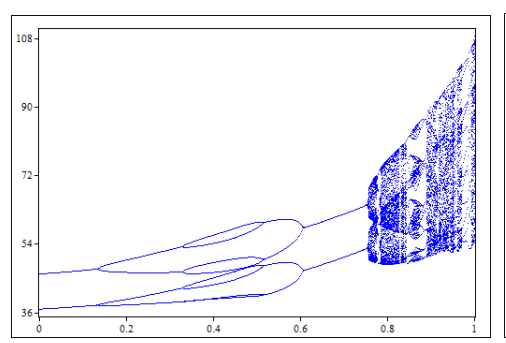

(B)

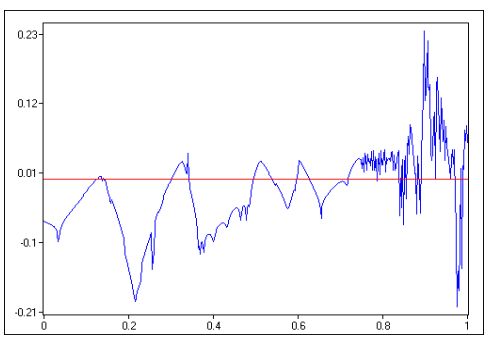

(C)

Figure 9: The bifurcation diagrams with respect to $\omega \in[0,1]$ for $P$ in $(\mathrm{A})$ and $Y$ in $(\mathrm{B})$, and the Lyapunov exponent in $(\mathrm{C})$, respectively, for $\mu=31$ and the initial conditions $P(0)=10, x(0)=0.5$ and $Y(0)=61$.

Summarizing, in the case of a stable financial market and an unstable real market, for the above parameter configurations we have been not able to find stabilizing values for $\omega$, even if intermediate values of $\omega$ may lead to a reduction of the complexity of the system. In this respect, in Figures 8 and 9 we highlight the presence of the so-called bubbles (see Hommes 1991, 1994). 


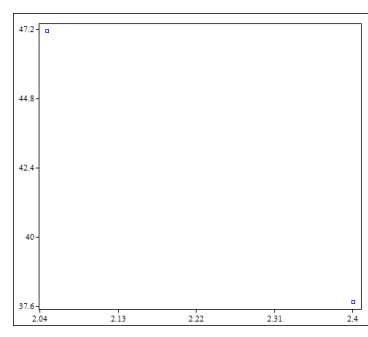

(A)

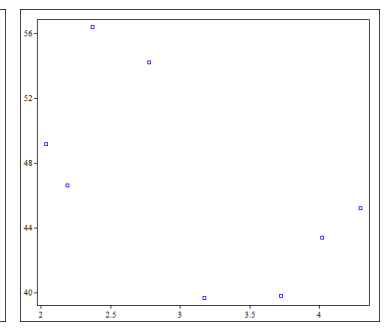

(B)

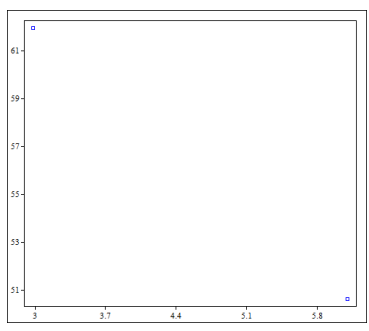

(C)

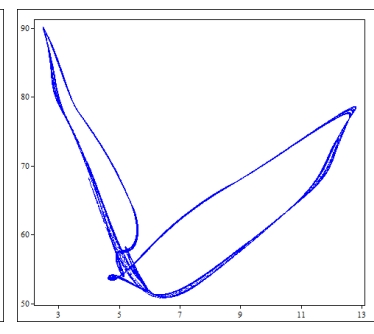

(D)

Figure 10: The $(P, Y)$-phase portrait for $\mu=31$ and $\omega=0.1$ in (A), $\omega=0.4$ in (B), $\omega=0.7$ in (C) and for $\omega=0.9$ in (D), respectively.

\subsection{Unstable financial and real subsystems}

In this last framework, when isolated, the financial and the real subsystems are unstable. In particular, when $\omega=0$ both of them may be chaotic. When $\omega$ increases, we did not find a complete stabilization of the system, but we notice some periodicity windows in Figures 11 and 12 below, where we have fixed the parameters as follows: $F^{*}=2, k=0.1, \alpha=0.08, \beta=1, c=1, a=2.4, \mu=28, \gamma=20, a_{1}=3, a_{2}=$ $1, A=12, b=0.7$. Notice that these are the same parameter values considered in the second scenario, except for a larger value of $\gamma$. We already noticed in Subsection 5.2 that increasing $\gamma$ has a destabilizing effect for the above parameter configuration and this is confirmed by Figures 11 and 12. More precisely, in Figures 11 (A) and (B) we show the bifurcation diagrams with respect to $\omega \in[0,1]$ for $P$ and $Y$, respectively; in Figure $11(\mathrm{C})$ we show the Lyapunov exponent when $\omega$ varies in $[0,1]$. In Figures $12(\mathrm{~A})-(\mathrm{D})$ we depict, in the $(P, Y)$-phase plane, the chaotic regime when $\omega=0$, a period-twelve cycle when $\omega=0.5$, a chaotic attractor when $\omega=0.8$ and a period-fourteen cycle when $\omega=1$, respectively.

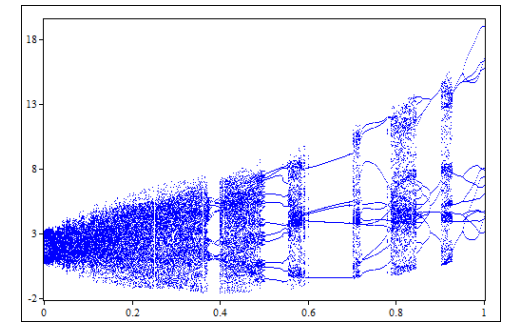

(A)

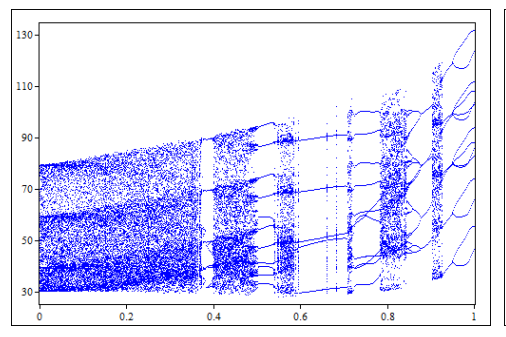

(B)

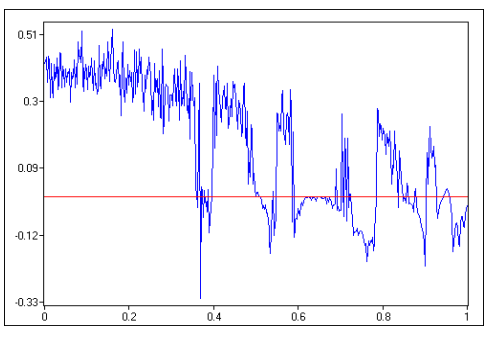

(C)

Figure 11: The bifurcation diagrams with respect to $\omega \in[0,1]$ for $P$ in $(\mathrm{A})$ and $Y$ in (B), and the Lyapunov exponent in (C), respectively, for the initial conditions $P(0)=6, x(0)=0.25$ and $Y(0)=0.63$. 


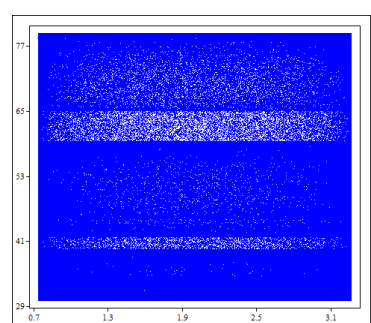

(A)

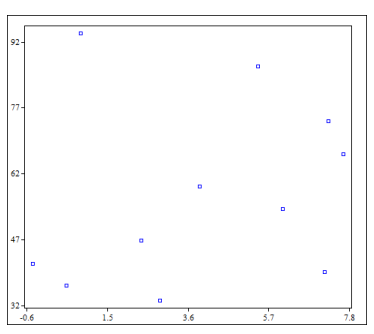

(B)

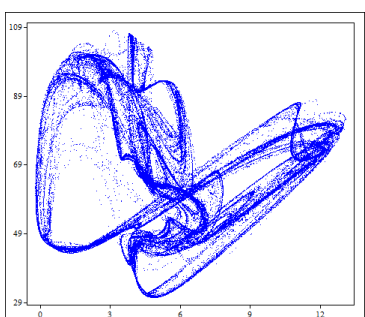

$(\mathrm{C})$

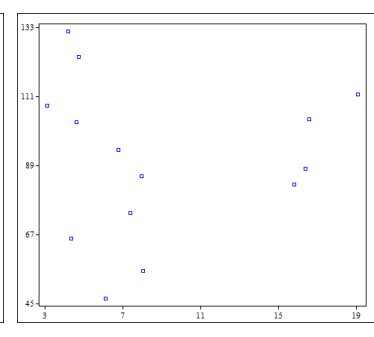

(D)

Figure 12: The $(P, Y)$-phase portrait for $\omega=0$ in $(\mathrm{A}), \omega=0.5$ in $(\mathrm{B}), \omega=0.8$ in $(\mathrm{C})$ and for $\omega=1$ in (D), respectively.

Let us now check whether the theoretical results in Section 4 are in agreement with the numerical achievements above.

First of all let us verify that, for the choice of the parameter set above, when $\omega=0$ neither the financial subsystem, nor the real subsystem are stable, i.e., let us check that neither the first chain of inequalities in (4.5), nor the last inequality therein are fulfilled. A straightforward computation shows that this is the case, as the first chain of inequalities reads as $0.116<1<0.968$ and the last inequality becomes $20<8.888$. As concerns the stability conditions when $\omega$ varies in $[0,1]$, we have $B_{1}=3, B_{2}=$ $0.024, B_{3}=0.038, B_{4}=-1.193$, and thus, since $B_{2}<B_{3}$, (4.2) implies that there exists no $\omega$ for which the system is stable, in agreement with Figures 11 and 12.

Summarizing, when both the financial and real markets are unstable, for the above parameter configuration (as well as for many others we investigated), we may have a reduction of the complexity until periodic motions, but not a complete stabilization of the system.

\subsection{The role of an increasing bias}

We conclude the present section by showing which are the effects of an increasing bias on the stability of the whole system. Since the results we got are uniform enough across the various scenarios considered so far, we illustrate our findings just for the first scenario, in which, when isolated, both the financial and the real subsystems are stable. Indeed, in Figure 13 below we have fixed the parameters as follows: $F^{*}=5$, $k=0.25, \alpha=0.08, \beta=1, c=1, \gamma=3.5, a_{1}=2, a_{2}=4, A=5, b=0.7, \omega=0.9$ and $\mu=5$ in Figure $13(\mathrm{~A})$, where we show the bifurcation diagram for $P$ with respect to $a \in[2,7]$, while $\mu=28$ in Figure $13(\mathrm{~B})$, where we depict the bifurcation diagram for $P$ with respect to $a \in[2,3.9]$. In Figure $13(\mathrm{~A})$ the steady state gets destabilized for $a \simeq 5.9$ through a Neimark-Sacker bifurcation. In Figure 13 (B) the steady state is instead unstable for values of $a$ close to the boundary of the considered interval, while it is stable for intermediate values of $a$.

Hence, even if the destabilizing role of the bias is clear when markets are iso- 


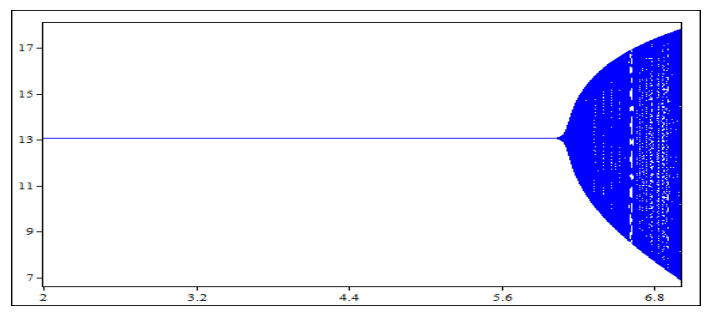

(A)

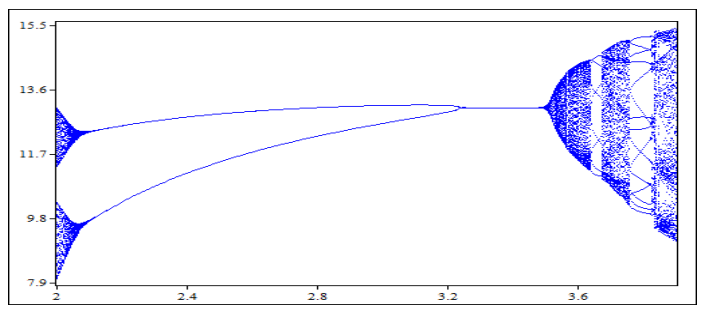

(B)

Figure 13: The bifurcation diagrams for $P$ with respect to $a \in[2,7]$ with $\mu=5$ in (A) and with respect to $a \in[2,3.9]$ with $\mu=28$ in (B), respectively, both obtained for the initial conditions $P(0)=10, x(0)=0.25$ and $Y(0)=50$.

lated (see (4.4)), Figure 13 suggests that its role becomes more ambiguous when the markets are interconnected. Indeed, increasing $a$ may have either a stabilizing or a destabilizing role, according to the value of the other parameters. However, taking into account also the conclusions we got for the other scenarios, it seems that increasing $a$ has generally a destabilizing effect, as usually we do not reach a complete stabilization, or we achieve it just in small intervals for the bias.

\section{Conclusion and future directions}

In the present paper we proposed a model belonging both to the strand of literature on the interactions between real and financial markets, as well as to the literature with heterogeneous fundamentalists. In fact, in the model we presented the real economy, described via a Keynesian good market approach, interacts with the stock market with heterogeneous speculators. More precisely, similarly to De Grauwe and Rovira Kaltwasser (2012), we assumed that the financial side is represented by a market where traders behave in two different ways: optimism and pessimism. However, differently from our setting, De Grauwe and Rovira Kaltwasser (2012) deal only with the financial sector, while the connection with the real side of the economy is missing. Moreover, similarly to Westerhoff (2012), we assumed the real economy to be represented by an income-expenditure model in which expenditures depend also on the dynamics of the stock market price. On the other hand, in Westerhoff (2012) the real market subsystem is described by a stable linear relation and the oscillating behavior is generated by the financial subsystem only. In our paper the oscillating behavior is generated instead also by the real subsystem.

We employed analytical and numerical tools in order to detect the mechanisms and the channels through which instabilities get transmitted between markets. The main contribution of the present paper to the existing literature lies in fact in our focus on the role of real and financial feedback mechanisms, not only in relation to the dynamics and stability of a single market, but for those of the economy as a whole. 
In order to perform such analysis, we introduced the "interaction degree approach", which allowed us to study the complete three-dimensional system by decomposing it into two subsystems, i.e., the isolated financial and real markets, easier to analyze, that are then interconnected through the "interaction parameter". We classified the possible scenarios according to the stability/instability of the isolated financial and real markets: in this way we have been led to analyze four frameworks. For each of those we considered different possible parameter configurations and we showed, both analytically and numerically, which are the effects of increasing the degree of interaction between the two markets. The conclusions we got are not univocal: indeed, depending on the value of the other parameters, an increase in the interaction parameter may either have a stabilizing or a destabilizing effect, but also other phenomena are possible. Nonetheless, comparing the results in the various scenarios, we could infer that the instability of the real market seems to have stronger destabilizing effects than the instability of the financial market: in fact, the former gets transmitted and possibly amplified by the connection with the financial market, while the latter gets dampened and possibly eliminated by the connection with the real market.

We concluded our analysis by showing which are the effects of an increasing bias. Although it is clearly destabilizing when markets are isolated, we found that its role becomes more ambiguous when the markets are interconnected. Indeed, increasing the bias may have either a stabilizing or a destabilizing role, according to the value of the other parameters. However, our numerical simulations suggested that increasing the bias has generally a destabilizing effect, as usually we did not reach a complete stabilization, or we achieved it just in small intervals for the corresponding parameter.

Future research should focus, for instance, on extending the model in order to include money and other financial assets. A different possible extension could concern the introduction in our model of chartists, in order to check whether they have a destabilizing effect also in the present context, or of unbiased fundamentalists, as already done in De Grauwe and Rovira Kaltwasser (2012), in view of comparing the results in the different scenarios. 


\section{References}

Anderson, S., de Palma, A., Thisse, J., 1992. Discrete Choice Theory of Product Differentiation. The MIT Press.

Brock, B., Hommes, C., 1997. A rational route to randomness. Econometrica 65, 1059-1095.

Brock, B., Hommes, C., 1998. Heterogeneous beliefs and routes to chaos in a simple asset pricing model. Journal of Economic Dynamics and Control 22, 1235-1274.

Charpe, M., Flaschel, P., Hartmann, F., Proaño, C., 2011. Stabilizing an unstable economy: Fiscal and monetary policy, stocks, and the term structure of interest rates. Economic Modelling 28, 2129-2136.

Chiarella, C., 1992. The dynamics of speculative behavior. Annals of Operations Research 37, 101-123.

Chiarella, C., He, X., 2002. Heterogeneous beliefs, risk and learning in a simple asset pricing model. Computational Economics 19, 95-132.

De Grauwe, P., 2012. Lectures on Behavioral Macroeconomics. Princeton University Press, New Jersey, USA.

De Grauwe, P., Grimaldi, M., 2006a. The Exchange Rate in a Behavioral Finance Framework. Princeton University Press, New Jersey, USA.

De Grauwe, P., Grimaldi, M., 2006b. Exchange rate puzzles: a tale of switching attractors. European Economic Review 50, 1-33.

De Grauwe, P., Rovira Kaltwasser, P., 2007. Modeling optimism and pessimism in the foreign exchange market. Working Paper 1962, CESifo.

De Grauwe, P., Rovira Kaltwasser, P., 2012. Animal spirits in the foreign exchange market. Journal of Economic Dynamics and Control 36, 1176-1192.

Diks, C., Dindo, P., 2008. Informational differences and learning in an asset market with boundedly rational agents. Journal of Economic Dynamics and Control $32,1432-1465$.

Farebrother, R.W., 1973. Simplified Samuelson conditions for cubic and quartic equations. The Manchester School 41, 396-400.

Frankel, J., Froot, K., 1986. Understanding the US dollar in the eighties: the expectations of chartists and fundamentalists. Economic Record 62, 24-38. 
Frankel, J., Froot, K., 1990. Chartists, fundamentalists and trading in the foreign exchange market. American Economic Review 80, 181-185.

Heitger, F., 2010. Asset Price and Wealth Dynamics with Heterogeneous ExpectationsA Dynamic Nonlinear Structural Model Approach. Ph.D. Thesis. University of Kiel.

Hommes, C., 1991. Adaptive learning and roads to chaos: The case of the cobweb. Economics Letters 26, 127-132.

Hommes, C., 1994. Dynamics of the cobweb model with adaptive expectations and nonlinear supply and demand. Journal of Economic Behavior and Organization $24,315-335$.

Hommes, C., 2013. Behavioral Rationality and Heterogeneous Expectations in Complex Economic Systems, Cambridge University Press, Cambridge.

Lengnick, M., Wohltmann, H.-W., 2013. Agent-based financial markets and New Keynesian macroeconomics: a synthesis. J. Econ. Interact. Coord. 2013, 1-32.

Lloyd, A.L., 1995. The Coupled Logistic Map: A Simple Model for the Effects of Spatial Heterogeneity on Population Dynamics. J. Theor. Biol. 173, 217-230.

Lux, T., Marchesi, M., 1999. Scaling and criticality in a stochastic multi-agent model of a financial market. Letters to Nature 397, 498-500.

Manzan, S., Westerhoff, F., 2005. Representativeness of news and exchange rate dynamics. Journal of Economic Dynamics and Control 29, 677-689.

Naimzada, A., Pireddu, M., 2013. Dynamic behavior of real and stock markets with a varying degree of interaction. Submitted.

Available at http://ssrn.com/abstract $=2294032$

Naimzada, A., Ricchiuti, G., 2008. Heterogeneous fundamentalists and imitative processes. Applied Mathematics and Computations 199, 171-180.

Naimzada, A., Ricchiuti, G., 2009. The dynamic effect of increasing heterogeneity in financial markets. Chaos, Solitons and Fractals 41, 1764-1772.

Rovira Kaltwasser, P., 2010. Uncertainty about fundamentals and herding behavior in the FOREX market. Physica A 389, 1215-1222.

Scheffknecht, L., Geiger, F., 2011. A behavioral macroeconomic model with endogenous boom-bust cycles and leverage dynamics. Discussion paper 37-2011, University of Hohenheim. 
Westerhoff, F., 2003. Expectations driven distortions in the foreign exchange market. Journal of Economic Behavior and Organization 51, 389-412.

Westerhoff, F., 2012. Interactions between the real economy and the stock market: A simple agent-based approach. Discrete Dynamics in Nature and Society 2012, Article ID 504840. 